\title{
Communication
}

\section{Fluorescence Micropatterning Based on the Polymeric Photobase Generator Containing Oxime-Urethane Groups by Dansylation}

\author{
Won San Choi ${ }^{\dagger}$, Hak Soo Kim, and Kyu Ho Chae* \\ Department of Polymer Engineering, Chonnam National University, Gwangju 500-757, Korea. \\ ${ }^{\dagger}$ Department of Chemical and Biological Engineering, Hanbat National University, Daejeon 307-719, Korea
}

\begin{abstract}
A polymeric photobase generator containing oxime-urethane groups is applied to a fluorescence micropatterning material. Polymer bearing oxime-urethane groups was prepared by copolymerization of methyl methacrylate with methacryloyloxyethyl benzophenoneoxime urethane (MBU). The reaction of amino groups in the irradiated copolymer film with dansyl chloride (Dns-Cl) was monitored by using UV absorption, IR absorption and fluorescence spectroscopy. The fluorescence spectrum of the $\mathrm{Dns}^{-} \mathrm{Cl}$ - treated irradiated copolymer film shows a strong fluorescence with a fluorescence maximum wavelength at $510 \mathrm{~nm}$. A blue fluorescent micropattern with a line width of $2 \sim 3 \mu \mathrm{m}$ was obtained. Treatment of the irradiated copolymer film with $\mathrm{Dns}^{-} \mathrm{Cl}$ and rhodamine $\mathrm{B}$ mixture led to the formation of green, red, and orange-colored fluorescence micropatterns. Thus, various colored micropatterns on a single polymer film can be obtained by selective excitation of each dye molecules.
\end{abstract}

In recent years, the formation of patterned functional images in thin polymer film has attracted considerable interest as a tool for the preparation of new functional materials as well as a subject of fundamental research in surface science. ${ }^{1-3}$ Especially, fluorescence patterning technology has attracted a great attention $^{4}$ because of its potential applicability in the area of photonic and electronic devices such as optical data storage systems and displays.

Several methods for the formation of fluorescent images have been developed. These include photoacid-catalyzed chemical amplification ${ }^{5-6}$ and photoacid-induced protonation. ${ }^{7-8}$ Such a generation of fluorescent images consists of selective removal of acid labile protecting groups by way of photochemical transformation followed by chemisorption of fluorescent dyes from solution into the patterned polymer film. Other methods such as photo-bleaching of fluorescent polymers, ${ }^{9-10}$ photo-splitting of cyclodimers of fluorescent precursors in polymers, ${ }^{11-12}$ and using polydiacetylene supramolecular systems in polymer films ${ }^{13}$ have also been reported.

On the other hand, photobase generators are a group of compounds that produce a base upon irradiation. It was reported that the photolysis of oxime-urethane groups led to the formation of amines, which induce a cross-linking reaction in an epoxy resin. $^{13}$ A polymeric photobase generator containing oxime-urethane groups has been used for preparation of image-recording materials. ${ }^{14-18}$ Here, we report fluorescence imaging based on the polymeric photobase generator containing oxime-urethane groups through the use of dansyl chloride (Dns-Cl), an amine-sensitive fluorescent dyes, in analogy with fluorescent image formation based on the photogenerated acids.

Scheme 1. Preparation of polymer containing oxime-urethane groups.
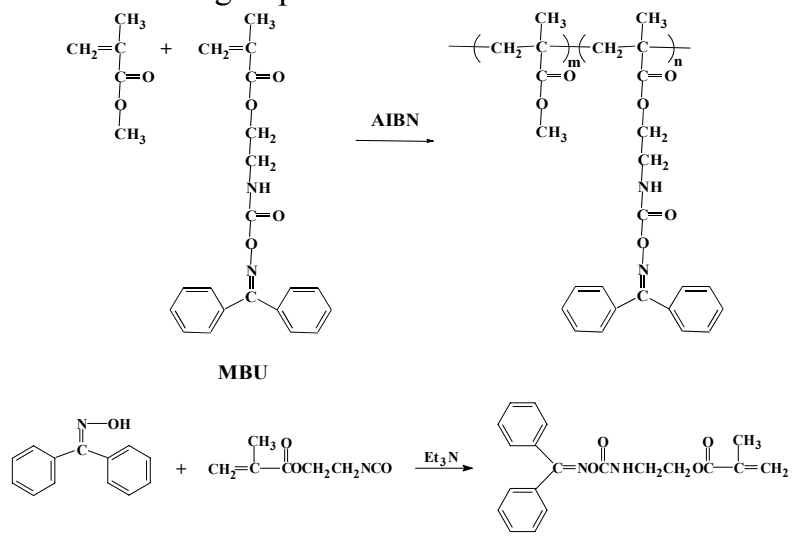

*To whom correspondence should be addressed.

E-mail: khochae@chonnam.ac.kr 
Table 1. Results of polymerization

\begin{tabular}{|c|c|c|c|c|c|c|}
\hline \multirow{2}{*}{ Copolymer } & \multicolumn{2}{|c|}{$\begin{array}{l}\text { Monomer } \\
\text { feed }(\mathrm{g})\end{array}$} & \multirow{2}{*}{$\begin{array}{l}\text { Mole \% } \\
\text { of } \\
\text { MBU } \\
\text { units in } \\
\text { feed }\end{array}$} & \multirow{2}{*}{$\begin{array}{l}\text { Mole\% of } \\
\text { MBU } \\
\text { units } \\
\text { found }\end{array}$} & \multirow{2}{*}{$M n$} & \multirow{2}{*}{$\begin{array}{c}T g \\
\left({ }^{\circ} \mathrm{C}\right)\end{array}$} \\
\hline & MBU & MMA & & & & \\
\hline I & 1.17 & 3.33 & 9.1 & 9.9 & 38000 & 81 \\
\hline II & 1.18 & 1.66 & 16.6 & 18.1 & 25000 & 81 \\
\hline
\end{tabular}

The copolymer containing oxime-urethane groups was prepared by polymerization of methyl methacrylate (MMA) and methacryloyloxyethyl benzophenoneoxime urethane (MBU) with azobisisobutyronitrile (AIBN) as an initiator as shown in Scheme 1.

MBU, a monomer containing oxime-urethane groups was prepared by the reaction of benzophenoneoxime and isocyanatoethyl methacrylate. Polymerization was carried out with $0.5 \mathrm{wt} \%$ of AIBN in $10 \mathrm{~mL}$ of THF at $60{ }^{\circ} \mathrm{C}$ for 20 hours. The copolymer is not soluble in alcohol or ether, but soluble in polar aprotic solvents. Table 1 shows results of copolymerization of the two monomers. The number of MBU units incorporated into polymer tends to be similar to that in the feed. The molecular weight of polymer II was lower than that of the polymer I due to the steric effect of benzophenoneoxime moiety in the MBU unit. A transparent and brittle film was obtained.

Scheme 2 shows the chemical reactions occur during the formation of the fluorescent images. A thin copolymer film is covered with a photomask and irradiated by UV light at $254 \mathrm{~nm}$. A latent image is formed in the irradiated area through the formation of amino groups. The amino groups are produced by the photo-decomposition of the oxime-urethane groups on the polymer surface. A fluorescent image is formed in the irradiated area through the reaction of the amino groups with Dns-Cl. The unreacted dye molecules on the polymer surface are washed out in the second stage of development.

\section{Scheme 2.}

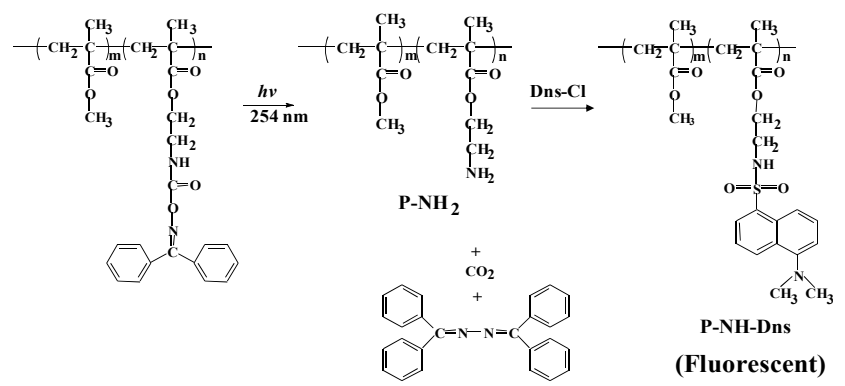

The photochemical reaction of the copolymer film containing oxime-urethane groups was studied by examining the changes in the UV absorption spectra upon irradiation. Figure 1 shows UV-vis absorption spectral changes of a copolymer I film upon irradiation with 254 nm UV light at various irradiation times. The absorption band at around 240-280 $\mathrm{nm}$ decreased upon irradiation because of the photodecomposition of oxime-urethane groups. In contrast, the absorption band at around 290-380 $\mathrm{nm}$ increased upon irradiation because of the formation of the photolysis products.

The reaction of the amino groups in the irradiated copolymer film with Dns-Cl was monitored by the UV-vis absorption spectral changes. Figure 2 shows UV-vis absorption spectra of a copolymer II film on the quartz plate after irradiation, after dipping the irradiated film in Dns-Cl solution (20 mg of $\mathrm{Dns}^{-} \mathrm{Cl}$ in toluene), and after washing the dipped film with toluene for $10 \mathrm{sec}$. The absorption band of the copolymer film at $382 \mathrm{~nm}$ increased when the polymer film was dipped in $\mathrm{Dns}-\mathrm{Cl}$ solution. This is due to the reaction of amino groups, which was formed in the irradiated copolymer film with Dns-Cl. The absorption band at $382 \mathrm{~nm}$ decreased when the dipped film was washed with toluene, since the unreacted Dns $\mathrm{Cl}$ molecules were removed.

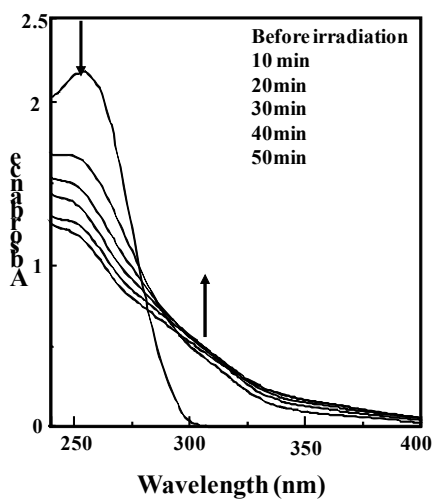

Figure 1. UV-vis absorption spectral changes of a copolymer II film on the quartz plate upon irradiation with $254 \mathrm{~nm}$ UV light at various irradiation times.

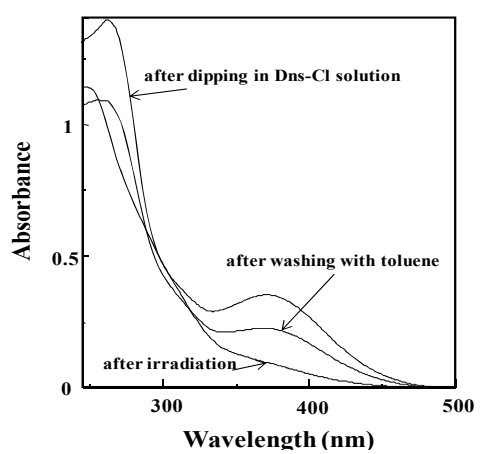

Figure 2. UV-vis absorption spectral changes of a copolymer II film on the quartz plate after irradiation, after dipping the irradiated film with $\mathrm{Dns}^{-} \mathrm{Cl}$ solution, and after washing the dipped film with toluene. 


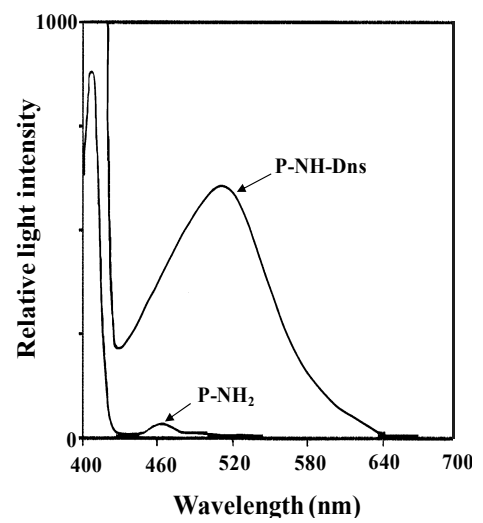

Figure 3. Fluorescence spectral changes of a copolymer II film on the quartz plate after irradiation $\left(\mathrm{P}-\mathrm{NH}_{2}\right)$ and after dipping the irradiated film in 25 $\mathrm{mM}$ Dns-Cl solution (P-NH-Dns).

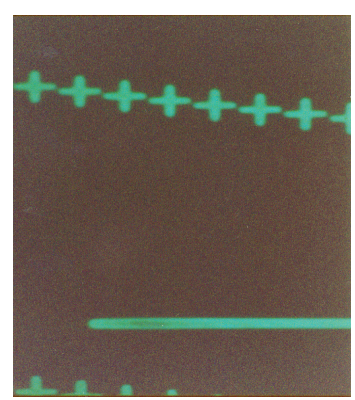

X 200

Figure 4. A fluorescent image obtained with a copolymer II film on the silicon wafer by dansylation. Exposure dose: $8.1 \mathrm{~J} / \mathrm{cm}^{2}$ at $254 \mathrm{~nm}$.

The dansylated copolymer film was observed by using IR absorption spectroscopy. A new absorption band at 1371 and $1175 \mathrm{~cm}^{-1}$ appeared after dansylation of the irradiated copolymer film, indicating the formation of $\mathrm{SO}_{2}$ groups. The new absorption bands at 698, 621, and $576 \mathrm{~cm}^{-1}$ also indicate the chemical reaction with dansyl groups.

Figure 3 shows fluorescence spectra of a copolymer II film on the quartz plate after irradiation with 254 $\mathrm{nm}$ UV light $\left(\mathrm{P}-\mathrm{NH}_{2}\right)$ and that after irradiation followed by dipping the irradiated film in a Dns-Cl solution. The irradiated copolymer film has no appreciable fluorescence, but it becomes fluorescent after the reaction with $\mathrm{Dns}^{-} \mathrm{Cl}$ with a fluorescence maximum wavelength at $510 \mathrm{~nm}$.

The absorption intensity of the dansylated copolymer film at $382 \mathrm{~nm}$ and emission intensity at $510 \mathrm{~nm}$ were affected by the various reaction conditions. In order to optimize the reaction conditions, the effect of reaction temperature, reaction time, and concentration of $\mathrm{Dns}^{-\mathrm{Cl}}$ on the absorbance of the dansylated copolymer film at $382 \mathrm{~nm}$ or fluorescence intensity at $510 \mathrm{~nm}$ was observed. It was revealed that the optimum reaction conditions are $25 \mathrm{mM}$ Dns-Cl concentration, $6 \mathrm{~min}$ of dipping time, and $50{ }^{\circ} \mathrm{C}$ of reaction temperature.

A thin polymer film on a silicon wafer was covered with a photomask and irradiated with $254 \mathrm{~nm}$ UV light. A latent image was formed in the irradiated area. The irradiated copolymer II film wasdipped in a $25 \mathrm{mM}$ Dns- $\mathrm{Cl}$ solution (20 mg in $3 \mathrm{~mL}$ toluene) at $50{ }^{\circ} \mathrm{C}$ for $6 \mathrm{~min}$, and then the dipped film was washed with toluene for $10 \mathrm{sec}$ to remove the unreacted Dns-Cl. Figure 4 shows an example of a fluorescent micropattern which was observed by using a fluorescence microscope. A stable, blue fluorescent micropattern with a line width of $2 \sim 3 \mu \mathrm{m}$ was obtained.

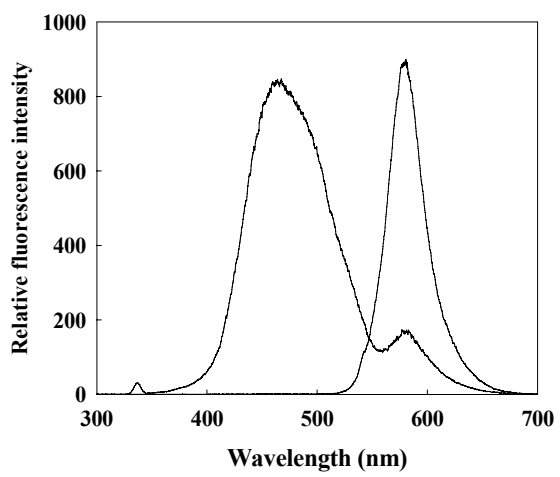

Figure 5. Fluorescence spectra of Dns-Cl and rhodamine $\mathrm{B}$ solution mixure $(1: 3, \mathrm{v} / \mathrm{v})$ at the excitation wavelength of 350 and $540 \mathrm{~nm}$.
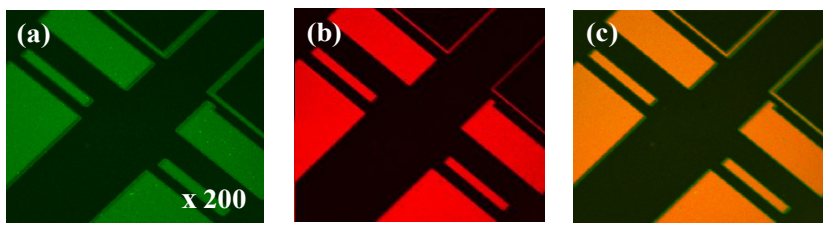

Figure 6. Fluorescent micropattern images of a copolymer II film on a silicon wafer observed by using a confocal laser scanning microscope (CLSM). Excitation wavelength: (a) $405 \mathrm{~nm}$, (b) $540 \mathrm{~nm}$, and (c) 405 and $540 \mathrm{~nm}$. The fluorescent images have been acquired at the wavelength range of (a) $450 \pm$ $25 \mathrm{~nm}$, (b) $590 \pm 25 \mathrm{~nm}$, and (c) $450 \pm 25$ and 590 $\pm 25 \mathrm{~nm}$. 
In order to obtain fluorescent different color images, a mixture of Dns-Cl and rhodamine B was used as fluorescent dyes. A mixture of $2.5 \mathrm{mM}$ Dns- $\mathrm{Cl}$ in toluene and $0.1 \mathrm{mM}$ rhodamine $\mathrm{B}$ in methanol $(1: 3 \mathrm{v} / \mathrm{v})$ was used as a fluorescence dye solution. Figure 5 shows fluorescence spectra of a Dns-Cl and rhodamine B solution mixture at the excitation wavelength of 350 and $540 \mathrm{~nm}$. Depending on the excitation wavelength, each molecule can be selectively excited. Dns-Cl was excited at the excitation wavelength of $350 \mathrm{~nm}$ with the emission maximum wavelength of $477 \mathrm{~nm}$, while rhodamine B was excited at the excitation wavelength of $540 \mathrm{~nm}$ with the emission maximum wavelength of $589 \mathrm{~nm}$.

A thin copolymer II film on a silicon wafer was covered with a photomask and irradiated. The latent image on the copolymer film was dipped in a $\mathrm{Dns}^{-\mathrm{Cl}}$ and rhodamine $\mathrm{B}$ solution mixture for $20 \mathrm{sec}$ and washed in toluene and methanol mixture $(1: 1, \mathrm{v} / \mathrm{v})$ for 30 sec. The dyed film was dried under reduced pressure at room temperature. The dyed image was observed by confocal microscopy. Differently colored fluorescent micropatterns are observed by the selective excitation of the each dye molecules.

Figure 6 shows an example of micropatterns obtained with a copolymer II film on a silicon wafer. A green fluorescent micropattern is observed on a dark background upon excitation at $405 \mathrm{~nm}$ with an observation wavelength range of $450 \pm 25 \mathrm{~nm}$. A red fluorescent micropattern on a dark background is observed upon excitation at $540 \mathrm{~nm}$ with an observation wavelength range of $590 \pm 25 \mathrm{~nm}$. An orange-colored fluorescent micropattern on a dark background is observed by simultaneously exciting at 405 and $540 \mathrm{~nm}$ when the observation wavelengths are $450 \pm 25$ and $590 \pm 25 \mathrm{~nm}$. Thus, different-colored fluorescent micropatterns are observed by varying the excitation wavelength.

We developed facile and easy strategies for the preparation of various colored micropatterns on the polymer film. Polymer containing oxime-urethane groups was synthesized and used for producing base moieties such as primary amines under UV irradiation, which can be further reacted with fluorescent dyes for patterning. The Dns-Cl-treated irradiated polymer film showed a strong green fluorescence. Our results also demonstrated that the color of micropatterns prepared with our approach can be tuned by appropriate combination and selective excitation of individual dyes. Additionally, our protocol allows for flexibility in material choice, such as the polymer films and dyes: thus, the characteristics of polymer films reacted with dyes can be controlled to suit various applications.
KEYWORDS: photofunctional polymer, fluorescence micro-patterning, photobase generator, oxime-urethane, dansylation, rhodamine $\mathrm{B}$.

Received March 21, 2013; Accepted April 20, 2013

\section{REFERENCES AND NOTES}

1. Campo, A.; Boos, D.; Spiess, H. W. Angew. Chem. Int. Ed. 2005, 44, 4707-4712 .

2. Zhang, X.; Zhang, J.; Ren, Z.; Zhang, X.; Tian, T.; Wang, Y.; Dong, F.; Yang, B. Nanoscale 2010, 2, 277-281.

3. Zhang, C.; Vekselman, A. M.; Darling, D. Chem. Mater. 1995, 7, 850-855.

4. Kim, J. M. Macromol. Rapid Commun., 2007, 28, 1191-1212.

5. Park, S.; Kim, S.; Seo, J; Park, S. Y. Macromolecules, 2005, 38, 4557-4559.

6. Yoo, J. H.; Kim, S. -Y.; Cho, I.; Kim, J. -M.; Ahn, K. -D.; Lee, J. H. Polymer 2004, 45, 5391-5395.

7. Kim, S; Park, S. Y. Adv. Mater. 2003, 15, 1341-1344.

8. Kim, J. M.; Chang, T. -E.; Kang, J. -H.; Han, D. K.; Ahn, K. -D. Adv. Mater. 1999, 11, 1499-1502.

9. Gao, L.; Lu, N.; Hao, J.; Hu, W.; Shi, G.; Wang, Y.; Chi, L.; Langmuir 2009, 25, 3894-3897.

10. Kocher, C.; Montali, A.; Smith, P.; Weder, C. Adv. Func. Mater. 2001, 11, 31-35.

11. Takahashi, K.; Takahashi, Y.; Yagai, S.; Kitamura, A.; Karatsu, T. J. Photopol. Sci. Tech. 2010, 23, 789-794.

12. Zhao, L.; Vaupel, M.; Loy, D. A.; Shea, K. J. Chem. Mater. 2008, 20, 1870-1876.

13. Chae, K. H. Macromol. Rapid Commun. 1998, 19, 1-4.

14. Chae, K. H.; Gwark, J. C.; Chang, T. Macromol. Rapid Commun. 2000, 21, 1007-1012.

15. Chae, K. H.; Jang, H. J. J. Polym. Sci., Part A: Polym. Chem. 2002, 40, 1200-1207.

16. Choi, W. S.; Noh, Y. -Y.; Chae, K. H. $A d v$. Mater. 2005, 17, 833-837.

17. Chae, K. H.; Kim, Y. H. Adv. Func. Mater. 2007, 17, 3470-3476.

18. Chae, K. H.; Baek, S. J. Macromol. Chem. Phys. 2012, 213, 1190-1195. 